\title{
Retraction Note to: shRNA-mediated EMMPRIN silencing inhibits human leukemic monocyte lymphoma U937 cell proliferation and increases chemosensitivity to adriamycin
}

\author{
Hui Gao ${ }^{1}$ Qixiao Jiang ${ }^{1} \cdot$ Yantao Han ${ }^{1} \cdot$ Jianjun Peng ${ }^{2} \cdot$ Chunbo Wang $^{1}$
}

Published online: 15 January 2022

(c) Springer Science+Business Media, LLC, part of Springer Nature 2022

Retraction Note to: Cell Biochem Biophys (2015) 71:827-835 https://doi.org/10.1007/s12013-014-0270-4

The Editor-in-Chief has retracted this article. After publication concerns were raised about overlap of Fig. 3B with Fig. 3C in [1] and of Fig. 4A with Fig. 3 in [1]. None of the authors has responded to correspondence about this retraction.

\section{Reference}

1. Gao, H., Xie, J., Peng, J., Han, Y., Jiang, Q., Han, M. \& Wang, C. (2015). Hispidulin inhibits proliferation and enhances chemosensitivity of gallbladder cancer cells by targeting HIF-1 $\alpha$. Experimental Cell Research, 332, 236-246.
Jianjun Peng

jianjunpeng@126.com

$\triangle$ Chunbo Wang cbwang666@126.com

1 Department of Pharmacology, Medical College, Qingdao, University, Qingdao 266021 Shandong, China

2 College of Life Sciences, Chongqing Normal University, Chongqing 401331, China 